\title{
A miRNA-based classification of renal cell carcinoma subtypes by PCR and in situ hybridization
}

\author{
Ashley Di Meo ${ }^{1,2,3}$, Rola Saleeb ${ }^{1,2}$, Samantha J. Wala ${ }^{1,2}$, Heba W. Khella ${ }^{1}$, Qiang \\ Ding ${ }^{1}$, Haiyan Zhai ${ }^{4}$, Krishan Kalra ${ }^{4}$, Adriana Krizova ${ }^{1}$, Manal Gabril ${ }^{5}$, Andrew \\ Evans $^{2}$, Fadi Brimo6, Maria D. Pasic ${ }^{2,7}$, Antonio Finelli ${ }^{8}$, Eleftherios P. Diamandis ${ }^{2,3}$ \\ and George M. Yousef ${ }^{1,2}$ \\ ${ }^{1}$ Department of Laboratory Medicine, Keenan Research Centre for Biomedical Science, Li Ka Shing Knowledge Institute, St. \\ Michael's Hospital, Toronto, ON, Canada \\ ${ }^{2}$ Department of Laboratory Medicine and Pathobiology, University of Toronto, Toronto, ON, Canada \\ ${ }^{3}$ Department of Pathology and Laboratory Medicine, Mount Sinai Hospital, Toronto, ON, Canada \\ ${ }^{4}$ BioGenex Laboratories, Fremont, CA, United States of America \\ ${ }^{5}$ Department of Pathology, London Health Sciences Center and Western University, London, ON, Canada \\ ${ }^{6}$ Department of Pathology, McGill University Health Centre, Montreal, QC, Canada \\ ${ }^{7}$ Department of Laboratory Medicine, St. Joseph's Health Centre, Toronto, ON, Canada \\ ${ }^{8}$ Division of Urologic Oncology, Princess Margaret Hospital, University Health Network, Department of Surgery, University of \\ Toronto, Toronto, ON, Canada
}

Correspondence to: George M. Yousef, email: yousefg@smh.ca

Keywords: renal oncocytoma; miRNA; precision medicine; renal cell carcinoma; in situ hybridization

Received: July 10, 2017 Accepted: November 15, 2017 Published: December 08, 2017

Copyright: Di Meo et al. This is an open-access article distributed under the terms of the Creative Commons Attribution License 3.0 (CC BY 3.0), which permits unrestricted use, distribution, and reproduction in any medium, provided the original author and source are credited.

\section{ABSTRACT}

Renal cell carcinoma ( $R C C)$ constitutes an array of morphologically and genetically distinct tumors the most prevalent of which are clear cell, papillary, and chromophobe RCC. Accurate distinction between the typically benign-behaving renal oncocytoma and RCC subtypes is a frequent challenge for pathologists. This is critical for clinical decision making. Subtypes also have different survival outcomes and responses to therapy. We extracted RNA from ninety formalin-fixed paraffinembedded (FFPE) tissues ( 27 clear cell, 29 papillary, 19 chromophobe, 4 unclassified RCC and 11 oncocytomas). We quantified the expression of six miRNAs (miR-221, miR-222, miR-126, miR-182, miR-200b and miR-200c) by qRT-PCR, and by in situ hybridization in an independent set of tumors. We developed a two-step classifier. In the first step, it uses expression of either miR-221 or miR-222 to distinguish the clear cell and papillary subtypes from chromophobe RCC and oncocytoma (miR-221 AUC: $0.96,95 \%$ CI: $0.9132-1.014, p<0.0001$ and miR-222 AUC: $0.91,95 \%$ CI: $0.8478-0.9772, p<0.0001)$. In the second step, it uses miR-126 to discriminate clear cell from papillary RCC (AUC: $1, p<0.0001$ ) and miR-200b to discriminate chromophobe RCC from oncocytoma (AUC: 0.95, 95\% CI: 0.8933-1.021, $p<0.0001$ ). In situ hybridization showed a nuclear staining pattern. miR-126, miR-222 and miR200b were significantly differentially expressed between the subtypes by in situ hybridization. miRNA expression could distinguish RCC subtypes and oncocytoma. miRNA expression assessed by either PCR or in situ hybridization can be a clinically useful diagnostic tool to complement morphologic renal tumor classification, improving diagnosis and patient management. 


\section{INTRODUCTION}

Renal cell carcinoma (RCC) is the most prevalent malignancy of the adult kidney, accounting for $90 \%$ of all kidney cancers $[1,2]$. Worldwide, over 260, 000 cases of kidney cancer are diagnosed annually and up to 116,000 people die as a result of the disease [2]. RCC comprises a heterogeneous group of tumors with distinct genetic and molecular characteristics [3]. Clear cell RCC (ccRCC), papillary RCC (pRCC) and chromophobe RCC (chRCC) are the most common RCC subtypes. In addition, $\sim 4 \%$ of RCC cases are labelled "unclassified" [4]. Oncocytoma, a benign neoplasm of the kidney, is found in up to $25 \%$ of early stage tumors managed with surgery [5].

Characterization of RCC subtypes relies on distinct histopathological characteristics. Clear cell tumors are hypervasular and usually have abundant clear cytoplasm while papillary tumors display papillary architecture with fibrovascular cores containing variable amounts of foamy macrophages. Chromophobe tumors have distinct cell borders, voluminous reticular cytoplasm, and perinuclear halos. Oncocytoma is characterized by polygonal cells that have abundant eosinophilic cytoplasm in addition to uniform rounded nuclei. However, morphology is not always conclusive and overlapping features certainly exist with tumors characterized by clear cytoplasm, tubulopapillary architecture and/or eosinophilic cytoplasm having a broad differential diagnosis. For instance, the eosinophilic variants of chRCC and ccRCC can exhibit similar cytological features. In addition, hybrid oncocytic chromophobe tumors (HOCT) contain histological features from both chromophobe RCC and renal oncocytoma. Interobserver variability amongst pathologists has also been reported [6]. Moreover, unclassified RCC exhibits variable microscopic features that do not fit any defined subtype [7].

RCC subtypes exhibit different clinical behaviour, prognosis and response to therapy [8]. ccRCC is most likely to present at advanced stage, higher grade and metastatic disease. In fact, $20-30 \%$ of clear cell patients present with metastasis at diagnosis. Additionally, clear cell tumors have worse cancer-specific and overall survival (OS) compared to papillary and chromophobe RCC [9]. Although less aggressive, papillary RCC type II often presents at higher stage and grade [10] whereas chromophobe tumors are considered more indolent [11]. Accurate classification of RCC is also of significant clinical importance for guiding type-specific treatment strategies in the new era of targeted therapy.

More recently, molecular markers showed promise as adjuvant tools for accurate classification by complementing morphologic evaluation, especially in small biopsies where diagnostic materials can be limited [12]. Reports have shown the ability of molecular signatures to "biologically" classify RCC. For instance, the two subtypes of pRCC were shown to have distinct biological signatures [13]. miRNAs are short non-coding
RNA fragments that control gene expression by pairing to the 3'UTR of target messenger RNA (mRNA). The link between miRNAs and cancer is well established in literature as they have been shown to play a key role in tumorigenesis and aggressive behaviour of tumors [14]. miRNAs have gained recognition as promising diagnostic, prognostic and predictive RCC biomarkers [15-17]. They have a number of advantages. They are highly stable and can be extracted from body fluids and formalin-fixed paraffin embedded (FFPE) tissues [18]. Earlier reports, including ours, have shown that miRNAs are differentially expressed between RCC subtypes [19-21]. We previously identified 65 miRNAs that were able to accurately differentiate between normal kidney, ccRCC, pRCC, chRCC and renal oncocytoma [19].

In this study, we measured the expression of six miRNAs in ninety FFPE renal tumor samples by qRTPCR. We developed a miRNA classifier that is able to differentiate between ccRCC, pRCC, and chRCC as well as renal oncocytoma in two steps. miRNAs included in the classification scheme were then quantified in four cases of unclassified RCC. We also examined the diagnostic utility of our miRNAs by in situ hybridization in an independent set of 98 FFPE RCC tissues.

\section{RESULTS}

\section{miRNA expression can classify renal cell carcinoma subtypes}

In our previously published data [19], we developed a classifier that can distinguish major subtypes of kidney tumors by pairwise comparison of the expression of 40 miRNAs in fresh tissues in a maximum of four steps. In order to translate this discovery into the clinic using a smaller number of highly informative miRNAs that can be measured on FFPE tissues, we tested six miRNAs (miR221, miR-222, miR-126, miR-182, miR-200b, and miR200 c) with potential utility as discriminatory markers. Selection criteria included frequency of dysregulation among subtypes, significance of differential expression, fold change, and evidence in literature of involvement in tumorigenesis. We quantified the expression of these miRNAs in ninety FFPE renal tumor samples by qRT-PCR. Five of these miRNAs showed differential expression between ccRCC, pRCC, chRCC and renal oncocytoma. Unsupervised clustering shows that with minimal exceptions, species of each histological type can be clustered together based on the expression of five specific miRNAs (Figure 1A).

\section{A miRNA classification system for distinguishing renal cell carcinoma subtypes}

We developed a miRNA classification system able to differentiate the most common RCC subtypes and 
renal oncocytoma in two steps, as detailed in Figure 1B. In the first step, the expression of miR-221 and miR-222 were able to differentiate ccRCC and pRCC from chRCC and oncocytoma (Table 1). miR-221 was significantly upregulated in chRCC and oncocytoma compared to ccRCC and pRCC (4.49-fold change, $p=6.39 \times 10^{-10}$ ) (Figure 2A), and was able to discriminate between the two groups (AUC: 0.96, 95\% CI: 0.9132-1.014, $p<0.0001$ ) (Figure 2B). miR-222 levels were significantly elevated in chRCC and oncocytoma (3.15-fold change, $p=2.46 \times$ $10^{-7}$ ) (Figure 2C), and showed significant discriminatory ability between the subtype pairs (AUC: 0.91, 95\% CI: 0.8478 to $0.9772, p<0.0001$ ) (Figure $2 \mathrm{D}$ ). As outlined in Table 1, miR-221 correctly identified 83 of 86 cases $(96.5 \%)$ whereas miR-222 accurately predicted 73 of 86 cases $(84.9 \%)$. As illustrated in Figure 2C, expression of miR-222 did not allow for complete separation between the subtype pairs. Mislabeled tumors from both groups

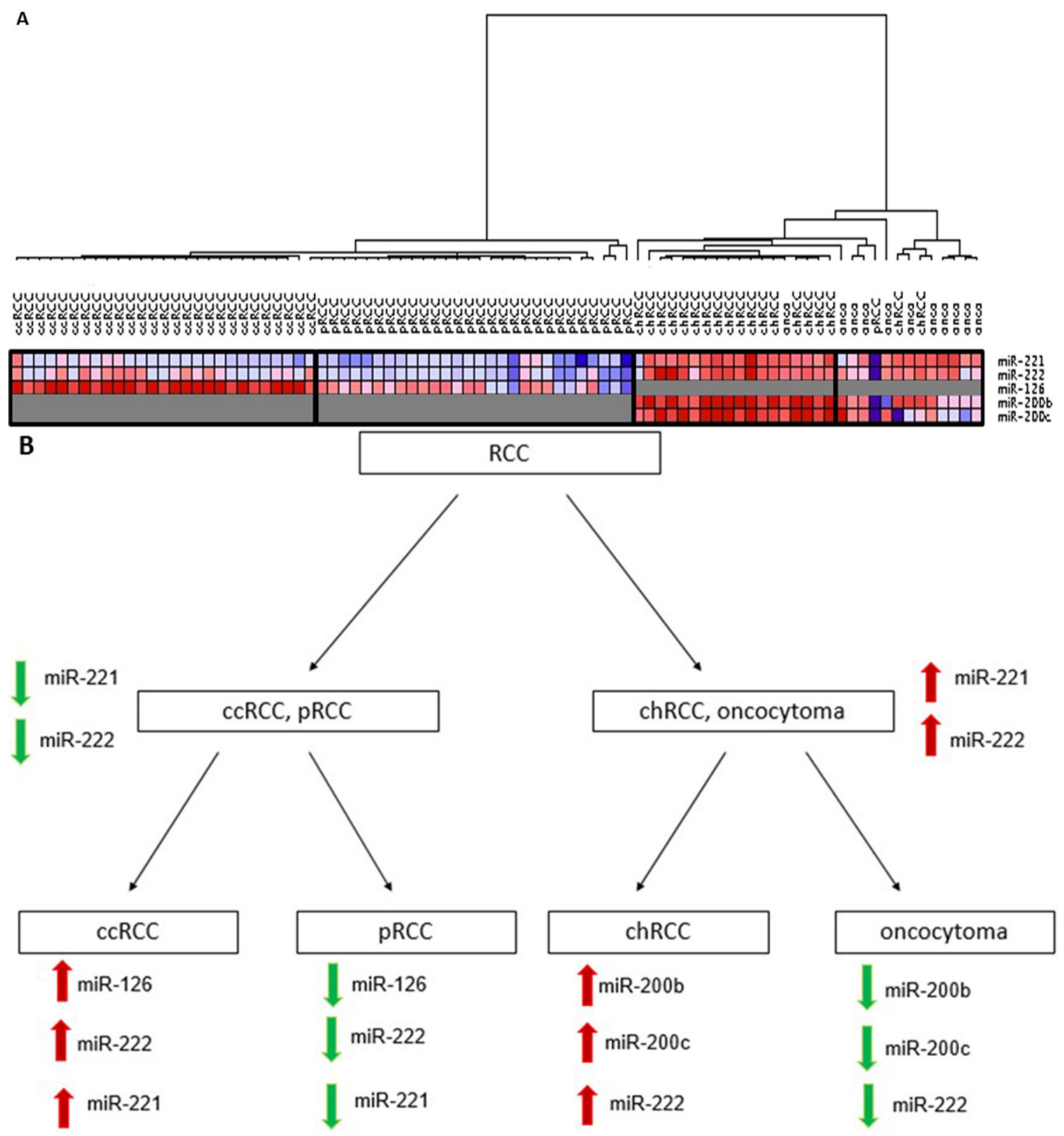

Figure 1: (A) Unsupervised hierarchical clustering of kidney tumors by miRNA expression. The samples clustered into four groups that closely follow the four histological types. Clear cell tumors (ccRCC) clustered closer to the papillary tumors (pRCC) whereas renal oncocytoma (onco) and chromophobe tumors (chRCC) clustered together. (B) Classification scheme for renal cell carcinoma subtypes and renal oncocytoma. First, samples are classified into either ccRCC and pRCC or chRCC and oncocytoma using expression of miR221 and miR-222. In the second step, ccRCC is differentiated from pRCC by miR-126, miR-222 and miR-221, and chRCC is differentiated from oncocytoma using expression of miR-200b, miR-200c and miR-222. ccRCC = clear cell renal cell carcinoma; $\mathrm{pRCC}=$ papillary $\mathrm{RCC}$; chRCC $=$ chromophobe RCC. 
Table 1: Expression of our miRNA classifiers in four histological types of renal tumors

\begin{tabular}{|c|c|c|c|c|c|c|c|c|c|c|c|c|c|c|c|c|}
\hline \multirow[b]{2}{*}{ miRNA } & \multirow[b]{2}{*}{ ccRCC } & \multicolumn{3}{|c|}{ Mean expression } & \multicolumn{4}{|c|}{$\begin{array}{c}\text { ccRCC + pRCC } \\
\text { versus } \\
\text { oncocytoma }+ \text { chRCC }\end{array}$} & \multicolumn{4}{|c|}{$\begin{array}{l}\text { ccRCC } \\
\text { versus } \\
\text { pRCC }\end{array}$} & \multicolumn{4}{|c|}{$\begin{array}{c}\text { Oncocytoma } \\
\text { versus } \\
\text { chRCC }\end{array}$} \\
\hline & & pRCC & Oncocytoma & $\operatorname{chRCC}$ & p-value & Fold & AUC & $\begin{array}{c}\text { Accuracy } \\
(\%)\end{array}$ & $p$-value & Fold & AUC & $\begin{array}{c}\text { Accuracy } \\
(\%)\end{array}$ & $p$-value & Fold & AUC & $\begin{array}{c}\text { Accuracy } \\
(\%)\end{array}$ \\
\hline miR-221 & 74.44 & 49.06 & 240.7 & 295.6 & $6.39 \times 10^{-10}$ & 4.49 & 0.96 & 96.51 & 0.0013 & 1.52 & 0.75 & 69.6 & 0.26 & 1.23 & 0.63 & 70 \\
\hline miR-222 & 707.2 & 312.9 & 947.5 & 1951 & $2.46 \times 10^{-7}$ & 3.15 & 0.91 & 84.88 & $3.47 \times 10^{-8}$ & 2.26 & 0.88 & 78.57 & 0.0002 & 2.1 & 0.87 & 83.33 \\
\hline miR-126 & 3203 & 308 & - & - & - & - & - & - & $1.24 \times 10^{-13}$ & 10.4 & 1 & 100 & - & - & - & - \\
\hline miR-200b & - & - & 85.93 & 283.6 & - & - & - & - & - & - & - & - & $1.75 \times 10^{-6}$ & 3.30 & 0.95 & 90 \\
\hline miR-200c & - & - & 150 & 503.8 & - & - & - & - & - & - & - & - & 0.002 & 3.36 & 0.82 & 83.33 \\
\hline
\end{tabular}

ccRCC = clear cell renal cell carcinoma; $\mathrm{pRCC}=$ papillary renal cell carcinoma; chRCC = chromophobe renal cell carcinoma.

Relative expression values.

fell into an overlapping "grey zone". In order to improve our biomarker performance, a 3-point cut-off was applied (Figure 2E). All cases were correctly identified as either chRCC or oncocytoma at a cut-off of $>1205$ copies whereas 37 of 38 cases $(97.4 \%)$ were correctly identified as either ccRCC or pRCC at a cut-off of $<589$ copies. All misclassified cases, except one, fell within the range of 589-1205 copies. The discriminatory ability of miR221 and miR-222 combined was less than miR-221 alone (AUC: $0.93, p<0.0001$ ).
In the second step, the expression of miR-126, miR182, miR-222 and miR-221 were tested to distinguish ccRCC from pRCC whereas miR-200b, miR-200c, miR222 and miR-221 were tested to differentiate chRCC from oncocytoma (Table 1). miR-126 was significantly overexpressed in ccRCC compared to pRCC (10.4-fold change, $p=1.24 \times 10^{-13}$ ) (Figure 3A), and was able to significantly discriminate between the two subtypes (AUC: $1, p<0.0001)$. It correctly classified all 56 cases $(100 \%)$ (Table 1, and Figure 3B). miR-222 was also significantly
A

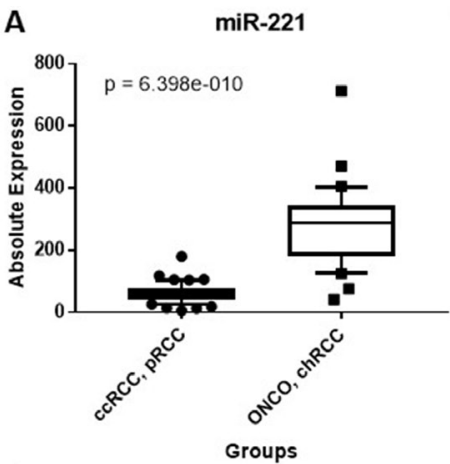

C

miR-222

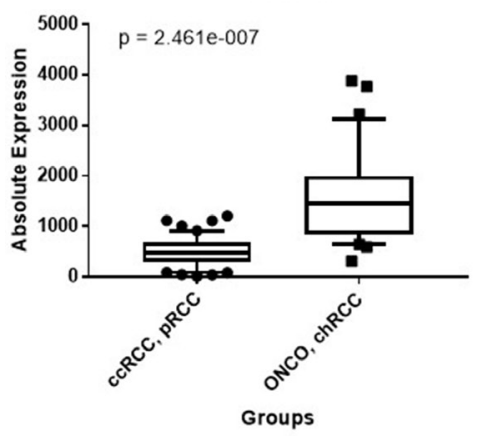

B

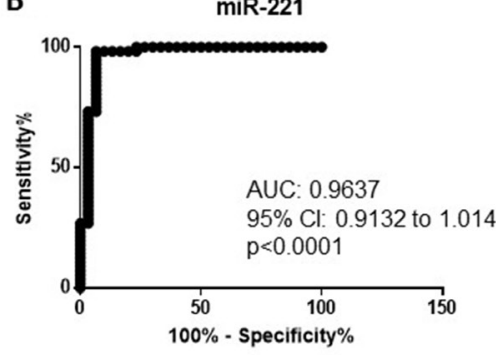

D

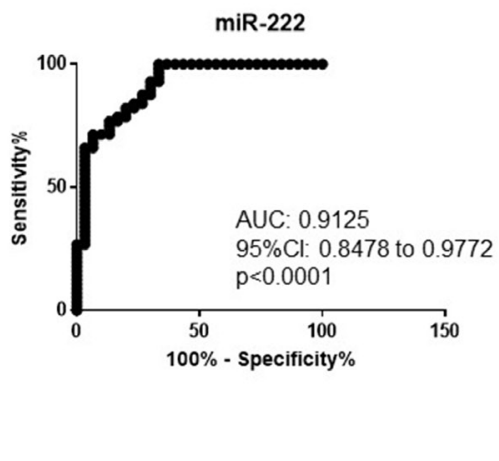

$\mathrm{E}$

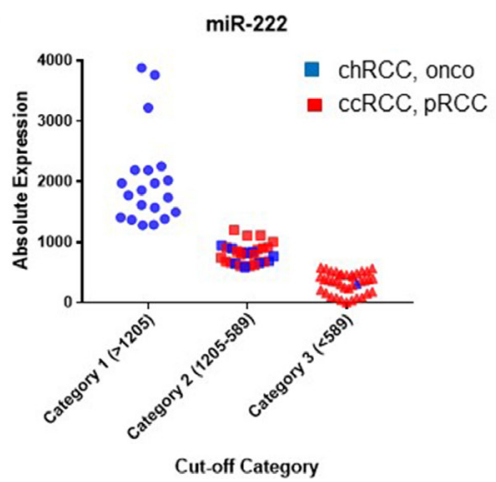

Figure 2: (A) miR-221 is significantly elevated in chRCC and renal oncocytoma relative to ccRCC and pRCC. (B) Receiver operating characteristic (ROC) curve showing the discriminatory ability of miR-221 to distinguish chRCC and renal oncocytoma from ccRCC and pRCC. (C) miR-222 is significantly overexpressed in chRCC and renal oncocytoma compared to ccRCC and pRCC. (D) ROC curve showing the discriminatory ability of miR-222 to distinguish the subtype pairs. Box plots (A and C) mean value (horizontal line), 10 to 90 percentile (box) and extent of data (whiskers). (E) Classification of tumors using a 3-point expression cut-off for miR-222. An expression cut-off was applied at $>1205$ copies (Category 1), between an expression range of 1205-589 copies (Category 2) and at $<589$ copies (Category 3 ). ccRCC = clear cell renal cell carcinoma; $\mathrm{pRCC}=$ papillary RCC; chRCC = chromophobe RCC; onco = oncocytoma. 
upregulated in ccRCC (2.26-fold change, $\left.p=3.47 \times 10^{-8}\right)$ (Figure 3C), although some cases were still overlapping (AUC: $0.88,95 \%$ CI: 0.799 to $0.966, p<0.0001$ ) (Figure 3D). When a 3-point cut-off was applied (Figure 3E), all cases were correctly identified as ccRCC at a cut-off of $>682$ copies whereas all cases were accurately identified as pRCC at a cut-off of $<358$ copies. Cases that were not accurately classified had expression levels between 682-358 copies. The expression of miR-221 was elevated in ccRCC relative to $\mathrm{pRCC}(1.51$-fold change, $p=0.0012)$ (Figure $3 \mathrm{~F}$ ), and had much less discriminatory power (AUC: 0.75 , 95\% CI: 0.6274 to $0.8847, p=0.001$ ) (Figure $3 \mathrm{G}$ ). miR222 correctly predicted 44 of 56 cases $(78.6 \%)$ whereas expression of miR-221 correctly classified 39 of 56 cases $(69.6 \%)$ as either ccRCC or pRCC, as shown in Table 1. The expression of miR-182 could not differentiate ccRCC from pRCC (data not shown). The discriminatory ability of miR-221 and miR-222 combined was less than miR-126 alone (AUC: $0.88,95 \%$ CI: 0.7954 to $0.9645, p<0.0001$ ).

The absolute expression of miR-200b was significantly higher in chRCC relative to oncocytoma (3.30-fold change, $p=1.75 \times 10^{-6}$ ) (Table 1 and Figure 4A). As illustrated in Figure 4B, miR-200b was able to distinguish chRCC from renal oncocytoma (AUC: 0.95, $95 \% \mathrm{CI}: 0.8933$ to $1.021, p<0.0001$ ) with a sensitivity of $89.5 \%$ and a specificity of $90.9 \%$ at a cut-off of 197 copies. miR-200b correctly identified 27 of 30 cases $(90 \%)$ as either chRCC or renal oncocytoma. miR-200c was significantly upregulated in chRCC (3.36-fold change, $p=0.0026$ ) (Table 1 and Figure 4C), and was able to discriminate between the two subtypes (AUC: 0.82, 95\% CI: 0.6361 to $1.01, p=0.0037)$ with $84 \%$ sensitivity and $82 \%$ specificity at a cut-off 180 copies (Figure 4D). It correctly classified 25 of 30 cases (83\%). miR-222 was also upregulated in chRCC compared to renal oncocytoma although to a lesser degree (2.1-fold change, $p=0.0002)$ (Figure 4E), and had a lower sensitivity relative to miR200b and miR-200c (AUC: 0.87, 79 \% sensitivity and 91\% specificity at a cut-off 1399 copies) (Figure 4F). miR-222 accurately classified 25 of 30 cases (83\%). Although miR221 was elevated in chRCC relative to renal oncocytoma, this did not reach statistical significance (Table 1). The discriminatory ability of miR-200b and miR-200c combined was less than miR-200b alone. This was also seen for the combination of miR-222 and miR-200b or miR-200c (data not shown).

\section{miRNA expression can help stratify unclassified RCC}

We tested the ability of miR-221, miR-222, miR126, miR-200b and miR-200c, which were included in the classification scheme, to stratify unclassified RCC. Sample A had extensive necrosis with few viable areas. Microscopic and immunohistochemical evaluation was inconclusive as the tumor displayed overlapping morphology of both clear cell and papillary subtypes (Supplementary Figure 2). Sample B displayed overlapping morphology of an eosinophilic RCC. Immunostaining was non-contributory. Sample $\mathrm{C}$ displayed variable morphologic types of renal cell carcinoma with areas of sarcomatoid differentiation, and sample D had mixed morphology.

As illustrated in Supplementary Figure 3A-3B), samples A, C and D classified as ccRCC or pRCC by miR-221 and miR-222 expression whereas sample B classified as chRCC or renal oncocytoma. Sample $\mathrm{A}$ and $\mathrm{C}$ were favoured to be pRCC whereas Sample D classified as ccRCC by miR-126 expression, as seen in Supplementary Figure 3C. Sample A was further confirmed as pRCC by miR-222 expression whereas samples $\mathrm{C}$ and $\mathrm{D}$ were inconclusive because their expression fell within the grey zone of miR-222 expression (between 682-358 copies, Supplementary Figure 3D). Samples A, C and D could not be accurately classified by miR-221 expression (data not shown). Sample B was further suggested to be renal oncocytoma using both miR-200b and miR-200c expression, as seen in Supplementary Figure 3E-3F), respectively, while using miR-222 expression, it was favoured to be chRCC (Supplementary Figure 3G). The above findings suggest that our miRNA classifier can be used to supplement morphology in defining the nature of the tumor, and also highlight the difficulty in separating chRCC from oncocytoma as discrete entities.

\section{miRNA in situ hybridization in renal cell carcinoma subtypes}

We next examined the expression of miR-126, miR222 and miR-221, assessed through in-situ hybridization, can be valuable markers to differentiate between the three most common RCC subtypes and oncocytoma. Representative stating patterns are shown in Figure 5. Using nuclear expression of miR-126 as a dichotomous variable, $89 \%$ of ccRCC tumors were positive compared to only $11 \%$ of pRCC $(p<0.0001)$ (Table 2$)$. The same pattern was seen for miR-222, with $71 \%$ of ccRCC tumors positive compared to only $29 \%$ of pRCC tumors showing positive expression $(p=0.013)$. A similar trend was seen for nuclear miR-221 expression, however this did not reach statistical significance $(p=0.0536)$.

In addition, we assessed nuclear expression of miR200b, miR-200c and miR-222 as a dichotomous variable in chRCC and oncocytoma. miR-200b was positive in $92 \%$ of chRCC compared to only $8 \%$ oncocytoma cases $(p=0.009)$ (Table 3). A similar trend was seen for nuclear expression of miR-200C, with $73 \%$ positivity in chRCC compared to only $27 \%$ of oncocytoma cases, although this was not statistically significant $(p=0.064)$ (Table 3$)$. Nuclear miR-222 expression did not show statistically significant differences. 


\section{DISCUSSION}

RCC represents a heterogeneous disease that encompasses a spectrum of distinct subtypes. The ability to subclassify RCC is of clinical importance since each subtype is a genetically distinct tumor with varying clinical course, prognosis and potential response to treatment. Currently, routine subtype classification relies on H\&E morphology and immunohistochemistry, however this is not always conclusive due to inter-observer variability and overlapping morphology [6].

miRNAs, a class of small non-coding RNAs have gained recognition as promising biomarkers [22]. The specificity and stability of miRNAs in FFPE tissues has encouraged their use as markers to subclassify RCC [23]. In the current study, we investigated the utility of miRNAs as potential biomarkers to aid in classifying RCC. We developed a miRNA classification system that can distinguish between the most common RCC subtypes and renal oncocytoma in two steps based on the expression of five miRNAs. We found that miR-221 and miR-222 could significantly distinguish ccRCC and pRCC from chRCC and oncocytoma. This is consistent with previous studies that reported elevated miR-221 levels in chRCC and oncocytoma relative to ccRCC and pRCC [20]. Clear cell and papillary RCC are believed to arise from the proximal tubules, while chRCC and oncocytoma are from the distal tubules [24]. Our results align with this since miR-221 and miR-222 expression separated ccRCC and pRCC from chRCC and oncocytoma. miR-126, miR-222 and miR-221 were able to significantly differentiate between ccRCC and pRCC. Several studies have reported elevated expression of miR-126 in ccRCC compared to pRCC [20, 25]. miR-126 is commonly associated with angiogenesis [26], which is a much more prominent feature ccRCC, compared to pRCC [27].

Expression levels of miR-200b, miR-200c and miR-222 were found to significantly differentiate chRCC from renal oncocytoma, although miR-200b had better discriminating ability. This is consistent with several reports that found miR-200b $[20,25]$ and miR-200c [20, $28]$ to be significantly elevated in chRCC compared to oncocytoma. Of the miRNAs included in our classification system, miR-221 had the best diagnostic performance in discriminating ccRCC and pRCC from chRCC and renal oncocytoma. miR-126 and miR-200b had the best diagnostic performance in discriminating ccRCC from pRCC and chRCC from renal oncocytoma, respectively. Upon review of cases that were incorrectly predicted, some had mixed morphology and were re-classified as hybrid oncocytic chromophobe tumors (HOCT). Our study is in keeping with the recent trend suggesting that oncocytoma and chRCC occupy opposite ends of the same tumor continuum. The use of histology as the gold standard for classification represents a limitation for our study.
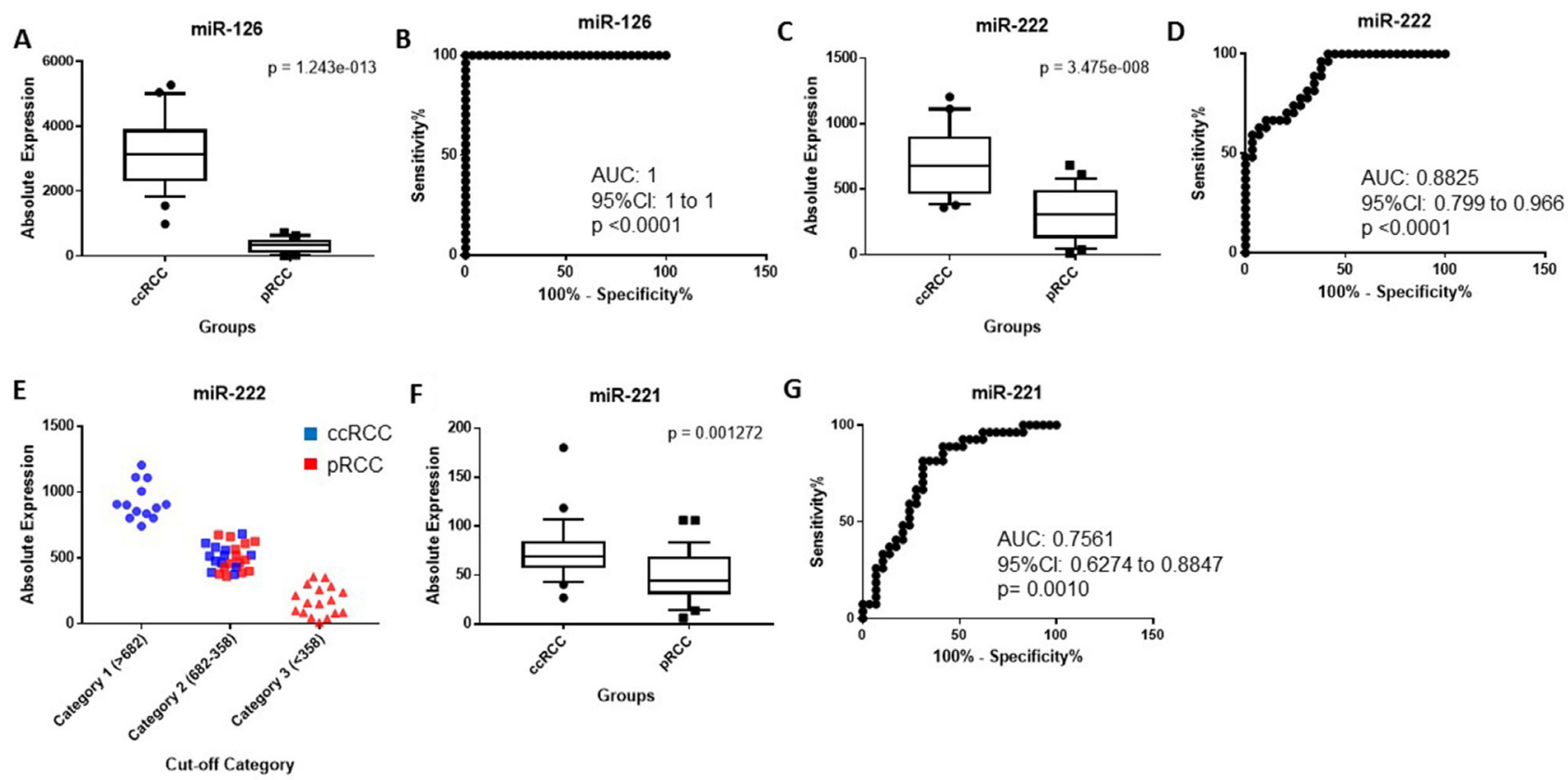

Figure 3: (A) miR-126 is significantly overexpressed in ccRCC compared to pRCC. (B) ROC curve showing the discriminatory ability of miR126 to discriminate ccRCC from pRCC. (C) miR-222 is significantly upregulated in ccRCC compared to pRCC. (D) ROC curve showing the discriminatory ability of miR-222 to distinguish the two groups. (E) Classification of ccRCC and pRCC using a 3-point expression cut-off for miR-222. An expression cut-off was applied at $>682$ copes (Category 1), between an expression range of 682-358 copies (Category 2) and at $<358$ copies (Category 3 ). (F) miR-221 is significantly elevated in ccRCC relative to pRCC. (G) ROC curve showing the discriminatory ability of miR-221 to discriminate ccRCC from pRCC. Box plots (A, C and E) mean value (horizontal line), 10 to 90 percentile (box) and extent of data (whiskers). ccRCC = clear cell renal cell carcinoma; $\mathrm{pRCC}=$ papillary $\mathrm{RCC}$. 
Table 2: Associations between nuclear miR-126, miR-222 and miR-221 expression, as dichotomous variable, and RCC patient diagnosis

\begin{tabular}{lcccc}
\hline Variable & total & \multicolumn{2}{c}{ No. of patients (\%) } & p-value \\
\cline { 3 - 3 } & & negative & positive & \\
\hline miR-126 & 28 & $24(85.71)$ & $4(10.81)$ & $<0.0001$ \\
pRCC & 37 & $4(14.29)$ & $33(89.19)$ & \\
ccRCC & & & $10(28.57)$ & 0.0134 \\
miR-222 & 28 & $18(60.00)$ & $25(71.43)$ & \\
pRCC & 37 & $12(40.00)$ & & \\
ccRCC & & & $16(34.78$ & 0.0536 \\
miR-221 & 28 & $12(63.16)$ & $30(65.22)$ & \\
pRCC & 37 & $7(36.84)$ & \\
ccRCC & &
\end{tabular}

ccRCC = clear cell renal cell carcinoma; $\mathrm{pRCC}=$ papillary $\mathrm{RCC}$

Bold refers to statistically significant values

Calculated using Fisher's Exact test.

Table 3: Associations between nuclear miR-200b, miR-200c and miR-222 expression, as dichotomous variable, and RCC patient diagnosis

\begin{tabular}{|c|c|c|c|c|}
\hline \multirow[t]{2}{*}{ Variable } & \multirow[t]{2}{*}{ total } & \multicolumn{2}{|c|}{ No. of patients $(\%)$} & \multirow[t]{2}{*}{$p$-valuc } \\
\hline & & negative & positive & \\
\hline \multicolumn{5}{|l|}{ miR-200b } \\
\hline $\operatorname{chRCC}$ & 20 & $9(42.86)$ & 11 (91.67) & 0.009 \\
\hline Oncocytoma & 13 & $12(57.14)$ & $1(8.33)$ & \\
\hline \multicolumn{5}{|l|}{ miR-200c } \\
\hline chRCC & 20 & $4(36.36)$ & $16(72.73)$ & 0.0645 \\
\hline Oncocytoma & 13 & $7(63.64)$ & $6(27.27)$ & \\
\hline \multicolumn{5}{|l|}{ miR-222 } \\
\hline $\operatorname{chRCC}$ & 20 & $5(71.43)$ & $15(57.69)$ & 0.6756 \\
\hline Oncocytoma & 13 & $2(28.57)$ & $11(42.31)$ & \\
\hline
\end{tabular}

chRCC = chromophobe RCC; onco = oncocytoma

Bold refers to statistically significant values

Calculated using Fisher's Exact test.

RCC subtypes exhibit characteristic chromosomal aberrations, such as whole or partial chromosomal duplications and deletions. Chromosomal alterations might explain, at least in part, the differential miRNA expression pattern observed among RCC subtypes. miR-221 and miR-222 are closely located on chromosome Xp11.3 [29]. Loss of chromosome $X$ was previously reported in pRCC [30]. This is consistent with our results, since miR222 expression was significantly lower in pRCC relative to ccRCC. miR-126 is located in the intronic region of the epidermal growth factor-like 7 gene (EGFL7) on chromosome 9 [31]. Frequent loss of chromosome 9p was also previously reported in pRCC [32], which may explain why expression levels of miR-126 are decreased in pRCC relative to ccRCC. Interestingly, loss of chromosome $9 p$ and $9 \mathrm{q}$ have also been reported in ccRCC [32], however this occurs at a later stage of disease progression [33].

Our study emphasizes the recent trend towards "molecular" classification of RCC. Brannon et al. identified two distinct ccRCC subtypes, designated clear cell type A (ccA) and B (ccB) [34]. Papillary tumors can also be subdivided into at least two subtypes, type I and II, based on distinct microscopic and gene expression patterns [35]. Oncocytic low-grade pRCC has also been described [36, 37]. These subtypes likely display distinct cytogenetic aberrations. For instance, chromosome 1p, 
$3 p$ and $9 p$ loss in addition to frequent gain of $5 q$ and $8 q$ are exclusively seen in type II [10] relative to papillary type I [13]. Oncocytic low-grade pRCC has chromosomal gains closer to type I pRCC [38], although more work is needed to assess the global cytogenetic aberrations of this tumor. Moreover, variants of chRCC exist, including an eosinophilic variant [39]. These variants may exhibit distinct molecular signatures. In fact, although classic chromophobe tumors show frequent loss of chromosomes $1,2,6,8,10,13,17$ and 21 , the eosinophilic variant was found to be mostly diploid [40].

miR-200b maps to chromosome $1 \mathrm{p} 36.33$ [21]. Several studies have reported high prevalence of chromosome 1 or $1 \mathrm{p}$ loss in renal oncocytoma [21, 41] , which would explain the lower miR-200b expression levels in oncocytoma. Moreover, miR-200c is located on chromosome 12 p13.31 [21]. Frequent copy number gains in chromosome 12 have been reported in chRCC [42]. Again, this is consistent with our results showing elevated miR-200c in chRCC.

Interestingly, select miRNAs were useful in pointing to distinct histological types in unclassified RCC, as seen above. Classification of RCC tumors can present a significant diagnostic challenge in cases where tumors display overlapping features [43]. Moreover, interobserver variability amongst pathologists can also make the differential diagnosis of tumors challenging [6]. Our results show a potential benefit of miRNA-based in situ hybridization assay using miRNA-specific probes in FFPE tissues to aid in the classification of common RCC subtypes. This can complement routine hematoxylin- eosin staining for improved RCC diagnosis. More detailed analysis of less-common subtypes are needed to further validate the performance of our markers.

We do acknowledge some limitations of our study. Independent validation is needed on larger case numbers. Also, we only focused on cases with classic morphology. We found that in situ hybridization showed less ability to accurately quantify miRNA expression levels compared to qRT-PCR analysis. Previous studies have shown that automated imaging algorithms are able to quantify staining intensity with high sensitivity [44, 45]. Thus, pairing chromogenic in situ hybridization to an automated imaging algorithm may increase the sensitivity of this platform.

A frequent struggle for pathologists is the accurate classification of incidentally detected small renal masses (pT1a, $\leq 4 \mathrm{~cm}$ ) on renal biopsy. Here, diagnostic material is often limited and morphology is in some cases inconclusive [46]. We speculate that our miRNA classifier may be useful for better classification of these cases on needle core biopsy. This would have significant implications for treatment of renal oncocytoma $\leq 4$ $\mathrm{cm}$, where active surveillance may be considered as an alternative option to immediate intervention [47]. In the clinical setting, qRT-PCR is feasible. It is possible to obtain ample amounts of RNA (0.5-1.3 $\mu \mathrm{g} / 20$ sections) from needle core biopsy [48]. Moreover, the qRT-PCR platform is also amenable to multiplexing which allows for quick turnaround. As mentioned above, chromogenic in situ hybridization can also be incorporated into routine hematoxylin-eosin staining of needle core biopsies for
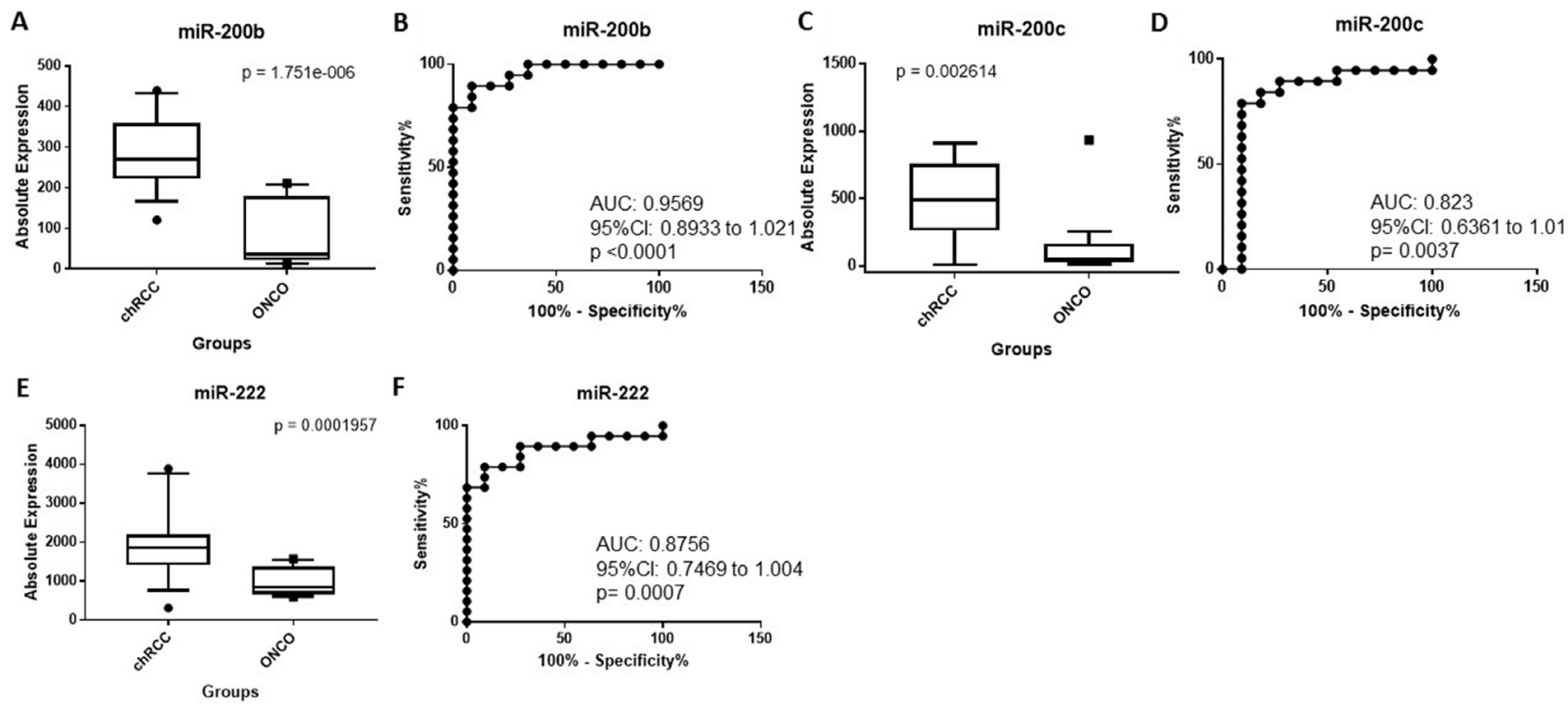

Groups

Figure 4: (A) miR-200b is significantly upregulated in chRCC compared to oncocytoma. (B) ROC curve showing the discriminatory ability of miR-200b to distinguish chRCC from oncocytoma. (C) miR-200c is significantly overexpressed in chRCC relative to oncocytoma. (D) ROC curve showing the discriminatory ability of miR-200c to distinguish chRCC from oncocytoma. (E) miR-222 is significantly elevated in chRCC relative to oncocytoma. (F) ROC curve showing the discriminatory ability of miR-222 to discriminate chRCC from oncocytoma. Box plots (A, C and E) mean value (horizontal line), 10 to 90 percentile (box) and extent of data (whiskers). chRCC $=$ chromophobe RCC; onco $=$ oncocytoma. 
improved classification of early-stage tumors.

Herein we demonstrate that the expression levels of a limited panel of miRNAs, including miR-221, miR-222, miR-126, miR-200b and miR-200c can help in subclassifying the most common RCC subtypes as well as renal oncocytoma. In addition, these miRNAs can provide insight into the molecular characteristics of individual unclassified tumors which may help in the selection of effective targeted therapies. Importantly, the miRNA classifier was validated by in situ hybridization, demonstrating the feasibility of this platform in classifying
RCC. To our knowledge, we are the first to assess the staining patterns of miR-221, miR-222, miR-126, miR$200 \mathrm{~b}$ and $\mathrm{miR}-200 \mathrm{c}$ by in situ hybridization for the differential diagnosis of RCC.

\section{MATERIALS AND METHODS}

\section{Specimen collection for RNA extraction}

For PCR analysis, we collected a total of 90 formalin-fixed paraffin-embedded RCC tissues from the

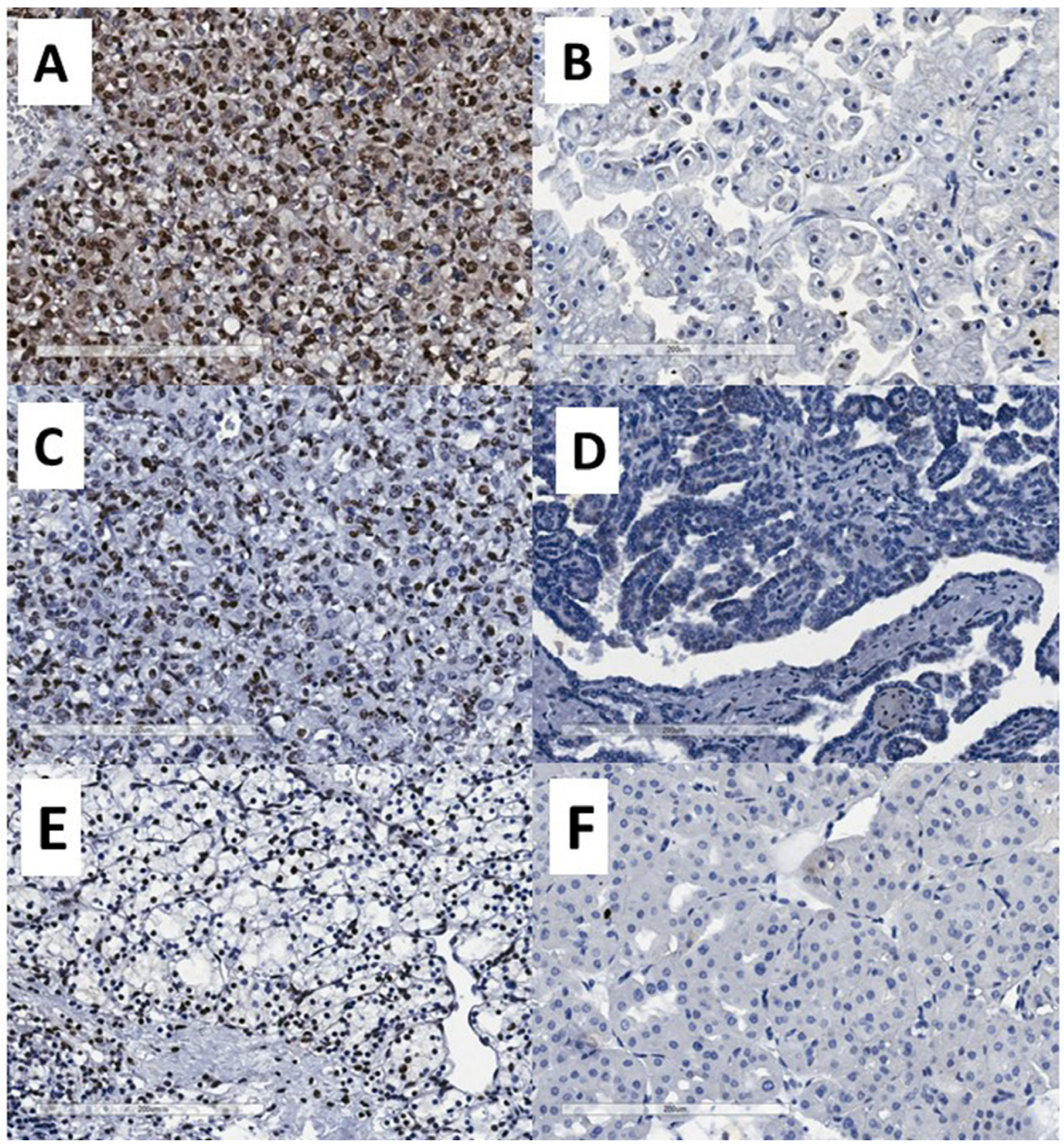

Figure 5: Representative photomicrographs showing miRNA expression by chromogenic in situ hybridization. (A) strong miR-126 nuclear expression, (B) weak/negative miR-126 nuclear expression, (C) strong miR-222 nuclear expression, (D) negative miR-222 nuclear expression, (E) strong miR-200b nuclear expression and (F) negative miR-200b nuclear expression. 
archives of the department of laboratory medicine at St. Michael's Hospital, Canada from 2002-2012 for qRT-PCR analysis. Diagnosis was reviewed according to the most recent Vancouver ISUP classification [4]. These included 27 ccRCC cases, 29 pRCC cases, 19 chRCC cases, 4 unclassified RCC cases and 11 oncocytoma cases. miRNA in situ hybridization was done on in an independent set of 98 FFPE RCC tissues.

\section{RNA extraction}

Sections of the formalin-fixed paraffin-embedded tissue corresponding to the designated subtype were selected for RNA extraction. Six cores of pure tumor tissue were obtained and pooled for each specimen to compensate for tumor heterogeneity. Tissues were taken from areas with no hemorrhage or necrosis. Total RNA was extracted using the miRNeasy FFPE Kit (Qiagen, Mississauga, Ontario) according to the manufacturer's protocol. RNA quality and concentration were determined spectrophotometrically (NanoDrop 2000c spectrophotometer, NanoDrop Technologies Inc., Wilmington, DE). Optimal RNA samples were stored at $-80^{\circ} \mathrm{C}$.

\section{miRNA quantification}

miRNA-specific reverse transcription was performed with $350 \mathrm{ng}$ total RNA using the TaqMan microRNA Reverse Transcription Kit (Applied Biosystems, Foster City, CA) as recommended by the manufacturer. RT-qPCR was performed using the TaqMan microRNA Assay Kit on Viia7 Real-Time PCR System (Applied Biosystems) [49] using miRNA-specific primers and probes. Thermal cycling conditions were according to the manufacturer's standard protocol and all reactions were performed in triplicates (Supplementary Figure 4). For generation of standard curves of chemically synthesized RNA oligonucleotides corresponding to miRNAs of interest, serial dilutions of each oligonucleotide were made in nuclease free water such that the final input into the RT reaction had a volume of 5 uL. Copy number was calculated using EndMemo (http:// www.endmemo.com/bio/dnacopynum.php). A line was fit to the data from each dilution series using $\mathrm{Ct}$ values within the linear range, from which $y=\operatorname{mln}(\times)+b$ equations were derived for quantification of absolute miRNA copies $(\times)$ from each sample $\mathrm{Ct}(\mathrm{y})$. A representative standard curve is illustrated in Supplementary Figure 1.

\section{Tissue microarray construction}

Tissue microarrays (TMAs) were built from 98 RCC formalin fixed paraffin-embedded tissue blocks from the surgical pathology archives of St. Michael's Hospital between 2001-2009. Diagnosis was confirmed by a pathologist according to the recent ISUP 2012 classification. These included 37 ccRCC cases, 28
pRCC cases, 20 chRCC cases and 13 oncocytoma cases. Each specimen was represented by two $1 \mathrm{~mm}$ cores obtained from two separate blocks to account for tumor heterogeneity. Areas of necrosis were avoided. Paraffin sections of the TMA were cut in 4 um thickness for in situ hybridization. The study was approved by the Research Ethics Board from St. Michael's Hospital, Toronto, Canada.

\section{In situ hybridization}

miRNA expression was assessed in FFPE TMA samples by in situ hybridization (ISH) performed by BioGenex Laboratories Inc. (Fremont, CA) with miRNA ISH probes and Super Sensitive One-step Polymer-HRP ISH detection kit on Xmatrx, the fully automated staining system (BioGenex Laboratories). In brief, these tissues were pre-treated with nucleic acid retrieval (NAR) solution at $85^{\circ} \mathrm{C}$ for 5 minutes and $100^{\circ} \mathrm{C}$ for 20 minutes. Then the tissues were incubated with hybridization buffer at $42^{\circ} \mathrm{C}$ for 20 minutes, followed by ISH using Fluorescein (FAM)-labeled miRNA probes for miR-200b, miR-200c, miR-126 and miR-222 $(100 \mathrm{nM})$ at $42^{\circ} \mathrm{C}$ for 2 hours. The non-specific binding probes were removed by stringent washes at $42^{\circ} \mathrm{C}$. The probes were detected by sequential addition of anti-fluorescein antibody followed by polyHRP. Final color visualization was performed by DAB along with hematoxylin counter staining.

\section{In situ hybridization scoring}

Hybridization signals were assessed semiquantitatively by two of the authors (A.D., R.S.)using a combined score resulting from summing the intensity of staining and frequency of immunoreactive cells. Staining intensity was 4-tier, including 0 (negative), 1 (weak), 2 (moderate), and 3 (strong). Frequency of immunoreactive cells was ranked in a 4-tier, including $0(0 \%), 1(<33 \%), 2$ (34\% to $66 \%$ ) and $3(>66 \%)$. Scores for miRNA staining were dichotomized into two groups; negative ( 0 to 2 ) and positive (3 to 6).

\section{Statistical analysis and clustering}

GraphPad Prism 7.1 and Perseus were used for statistical analysis. RT-PCR measurements $\left(C_{\mathrm{t}}\right.$ values) for 6 miRNAs were obtained for each specimen. $C_{t}$ values greater than 35 were considered to be non-expressed and truncated to 35. The unknown absolute expression level of each miRNA was interpolated from a semi-log curve. For every group (e.g. Oncocytoma versus chromophobe or clear cell versus papillary), absolute miRNA expression was compared for all miRNAs. The discriminatory power of miRNA combinations, calculated as a sum of absolute miRNA expression was also determined. $P$ values were calculated using a Welch's two-tailed Student's $t$-test. 
For differentially expressed miRNAs receiver operator characteristic (ROC) curves were constructed and the areas under the curve (AUC) were calculated to assess diagnostic performance. For in situ hybridization, Fisher's Exact Test was used to evaluate associations between miRNA staining pattern and RCC diagnosis. The Wilson-Brown method was used to compute confidence intervals (CI's), sensitivity and specificity. Hierarchical clustering was performed using GenePattern software (https://genepattern.broadinstitute.org, accessed November, 2016) [50, 51]. Absolute expression values were log-transformed and Pearson correlation was used to calculate the distance between every pair of samples.

\section{Abbreviations}

ccRCC; clear cell renal cell carcinoma, pRCC; papillary RCC, chRCC; chromophobe RCC, HOCT; hybrid oncocytic chromophobe tumors, ISH; in situ hybridization.

\section{Author contributions}

AD performed the majority of experimental work as well as data analysis and authored the manuscript. RM assisted with in situ hybridization analysis and participated in editing the manuscript. SJW and HWK assisted with experimental analysis and participated in editing the manuscript. $\mathrm{HZ}$ and $\mathrm{KK}$ assisted with the in situ hybridization experiments. $\mathrm{AK}, \mathrm{MG}, \mathrm{AE}$, and $\mathrm{FB}$ assisted in the clinical data analysis and participated in editing the manuscript. MDP, AF, and EPD participated in authoring and editing the manuscript. GMY coordinated the project and participated in authoring and editing the manuscript. All authors reviewed the results and approved the final version of the manuscript.

\section{ACKNOWLEDGMENTS}

Not applicable.

\section{CONFLICTS OF INTEREST}

The authors have declared no conflicts of interest.

\section{FUNDING}

This work was supported by grants from the Canadian Institute of Health Research (MOP 119606), Kidney Foundation of Canada (KFOC130030), and the Kidney Cancer Research Network of Canada.

\section{REFERENCES}

1. Siegel R, Ma J, Zou Z, Jemal A. Cancer statistics, 2014. CA Cancer J Clin. 2014; 64:9-29.
2. Ljungberg B, Campbell SC, Choi HY, Jacqmin D, Lee JE, Weikert S, Kiemeney LA. The epidemiology of renal cell carcinoma. Eur Urol. 2011; 60:615-621.

3. Bhatt JR, Finelli A. Landmarks in the diagnosis and treatment of renal cell carcinoma. Nat Rev Urol. 2014; 11:517-525.

4. Srigley JR, Delahunt B, Eble JN, Egevad L, Epstein JI, Grignon D, Hes O, Moch H, Montironi R, Tickoo SK, Zhou M, Argani P, and ISUP Renal Tumor Panel. The International Society of Urological Pathology (ISUP) Vancouver Classification of Renal Neoplasia. Am J Surg Pathol. 2013; 37:1469-1489.

5. Meloni-Ehrig AM. Renal cancer: cytogenetic and molecular genetic aspects. Am J Med Genet. 2002; 115:164-172.

6. Kummerlin I, ten Kate F, Smedts F, Horn T, Algaba F, Trias I, de la Rosette J, Laguna MP. Core biopsies of renal tumors: a study on diagnostic accuracy, interobserver, and intraobserver variability. Eur Urol. 2008; 53:1219-1225.

7. Tan PH, Cheng L, Rioux-Leclercq N, Merino MJ, Netto G, Reuter VE, Shen SS, Grignon DJ, Montironi R, Egevad L, Srigley JR, Delahunt B, Moch H, and ISUP Renal Tumor Panel. Renal tumors: diagnostic and prognostic biomarkers. Am J Surg Pathol. 2013; 37:1518-1531.

8. Nguyen DP, Vertosick EA, Corradi RB, Vilaseca A, Benfante NE, Touijer KA, Sjoberg DD, Russo P. Histological subtype of renal cell carcinoma significantly affects survival in the era of partial nephrectomy. Urol Oncol. 2016; 34:259 e251-258.

9. Leibovich BC, Lohse CM, Crispen PL, Boorjian SA, Thompson RH, Blute ML, Cheville JC. Histological subtype is an independent predictor of outcome for patients with renal cell carcinoma. J Urol. 2010; 183:1309-1315.

10. Klatte T, Pantuck AJ, Said JW, Seligson DB, Rao NP, LaRochelle JC, Shuch B, Zisman A, Kabbinavar FF, Belldegrun AS. Cytogenetic and molecular tumor profiling for type 1 and type 2 papillary renal cell carcinoma. Clin Cancer Res. 2009; 15:1162-1169.

11. Amin MB, Amin MB, Tamboli P, Javidan J, Stricker H, dePeralta Venturina M, Deshpande A, Menon M. Prognostic impact of histologic subtyping of adult renal epithelial neoplasms: an experience of 405 cases. Am J Surg Pathol. 2002; 26:281-291.

12. Arsanious A, Bjarnason GA, Yousef GM. From bench to bedside: current and future applications of molecular profiling in renal cell carcinoma. Mol Cancer. 2009; 8:20.

13. Wala SJ, Karamchandani JR, Saleeb R, Evans A, Ding Q, Ibrahim R, Jewett M, Pasic M, Finelli A, Pace K, Lianidou E, Yousef GM. An integrated genomic analysis of papillary renal cell carcinoma type 1 uncovers the role of focal adhesion and extracellular matrix pathways. Mol Oncol. 2015; 9:1667-1677.

14. White NM, Fatoohi E, Metias M, Jung K, Stephan C, Yousef GM. Metastamirs: a stepping stone towards improved cancer management. Nat Rev Clin Oncol. 2011; $8: 75-84$. 
15. Faragalla H, Youssef YM, Scorilas A, Khalil B, White NM, Mejia-Guerrero S, Khella H, Jewett MA, Evans A, Lichner Z, Bjarnason G, Sugar L, Attalah MI, Yousef GM. The clinical utility of miR-21 as a diagnostic and prognostic marker for renal cell carcinoma. J Mol Diagn. 2012; 14:385-392.

16. Nofech-Mozes R, Khella HW, Scorilas A, Youssef L, Krylov SN, Lianidou E, Sidiropoulos KG, Gabril M, Evans A, Yousef GM. MicroRNA-194 is a Marker for Good Prognosis in Clear Cell Renal Cell Carcinoma. Cancer Med. 2016; 5:656-664.

17. Khella HW, Butz H, Ding Q, Rotondo F, Evans KR, Kupchak P, Dharsee M, Latif A, Pasic MD, Lianidou E, Bjarnason GA, Yousef GM. miR-221/222 Are Involved in Response to Sunitinib Treatment in Metastatic Renal Cell Carcinoma. Mol Ther. 2015; 23:1748-1758.

18. Weber JA, Baxter DH, Zhang S, Huang DY, Huang KH, Lee MJ, Galas DJ, Wang K. The microRNA spectrum in 12 body fluids. Clin Chem. 2010; 56:1733-1741.

19. Youssef YM, White NM, Grigull J, Krizova A, Samy C, Mejia-Guerrero S, Evans A, Yousef GM. Accurate molecular classification of kidney cancer subtypes using microRNA signature. Eur Urol. 2011; 59:721-730.

20. Fridman E, Dotan Z, Barshack I, David MB, Dov A, Tabak $\mathrm{S}$, Zion O, Benjamin S, Benjamin H, Kuker H, Avivi C, Rosenblatt K, Polak-Charcon S, et al. Accurate molecular classification of renal tumors using microRNA expression. J Mol Diagn. 2010; 12:687-696.

21. Silva-Santos RM, Costa-Pinheiro P, Luis A, Antunes L, Lobo F, Oliveira J, Henrique R, Jeronimo C. MicroRNA profile: a promising ancillary tool for accurate renal cell tumour diagnosis. Br J Cancer. 2013; 109:2646-2653.

22. Khella HW, Bakhet M, Lichner Z, Romaschin AD, Jewett MA, Yousef GM. MicroRNAs in kidney disease: an emerging understanding. Am J Kidney Dis. 2013; 61:798-808.

23. Guo Z, Maki M, Ding R, Yang Y, Zhang B, Xiong L. Genome-wide survey of tissue-specific microRNA and transcription factor regulatory networks in 12 tissues. Sci Rep. 2014; 4:5150.

24. Shen SS, Krishna B, Chirala R, Amato RJ, Truong LD. Kidney-specific cadherin, a specific marker for the distal portion of the nephron and related renal neoplasms. Mod Pathol. 2005; 18:933-940.

25. Petillo D, Kort EJ, Anema J, Furge KA, Yang XJ, Teh BT. MicroRNA profiling of human kidney cancer subtypes. Int J Oncol. 2009; 35:109-114.

26. Khella HW, Scorilas A, Mozes R, Mirham L, Lianidou E, Krylov SN, Lee JY, Ordon M, Stewart R, Jewett MA, Yousef GM. Low expression of miR-126 is a prognostic marker for metastatic clear cell renal cell carcinoma. Am J Pathol. 2015; 185:693-703.

27. Douglas ML, Richardson MM, Nicol DL. Endothelin axis expression is markedly different in the two main subtypes of renal cell carcinoma. Cancer. 2004; 100:2118-2124.
28. Yoshino H, Enokida H, Itesako T, Tatarano S, Kinoshita T, Fuse M, Kojima S, Nakagawa M, Seki N. Epithelialmesenchymal transition-related microRNA-200s regulate molecular targets and pathways in renal cell carcinoma. $\mathrm{J}$ Hum Genet. 2013; 58:508-516.

29. Gottardo F, Liu CG, Ferracin M, Calin GA, Fassan M, Bassi P, Sevignani C, Byrne D, Negrini M, Pagano F, Gomella LG, Croce CM, Baffa R. Micro-RNA profiling in kidney and bladder cancers. Urol Oncol. 2007; 25:387-392.

30. Jiang F, Richter J, Schraml P, Bubendorf L, Gasser T, Sauter G, Mihatsch MJ, Moch H. Chromosomal imbalances in papillary renal cell carcinoma: genetic differences between histological subtypes. Am J Pathol. 1998; 153:1467-1473.

31. Zhang Y, Yang P, Sun T, Li D, Xu X, Rui Y, Li C, Chong M, Ibrahim T, Mercatali L, Amadori D, Lu X, Xie D, et al. miR-126 and miR-126 ${ }^{*}$ repress recruitment of mesenchymal stem cells and inflammatory monocytes to inhibit breast cancer metastasis. Nat Cell Biol. 2013; 15:284-294.

32. Kang XL, Zou H, Pang LJ, Hu WH, Zhao J, Qi Y, Liu CX, Hu JM, Tang JX, Li HA, Liang WH, Yuan XL, Li F. Chromosomal imbalances revealed in primary renal cell carcinomas by comparative genomic hybridization. Int $\mathrm{J}$ Clin Exp Pathol. 2015; 8:3636-3647.

33. Zhang Z, Wondergem B, Dykema K. A Comprehensive Study of Progressive Cytogenetic Alterations in Clear Cell Renal Cell Carcinoma and a New Model for ccRCC Tumorigenesis and Progression. Adv Bioinformatics. 2010:428325.

34. Brannon AR, Reddy A, Seiler M, Arreola A, Moore DT, Pruthi RS, Wallen EM, Nielsen ME, Liu H, Nathanson KL, Ljungberg B, Zhao H, Brooks JD, et al. Molecular Stratification of Clear Cell Renal Cell Carcinoma by Consensus Clustering Reveals Distinct Subtypes and Survival Patterns. Genes Cancer. 2010; 1:152-163. https:// doi.org/10.1177/1947601909359929.

35. Albiges L, Guegan J, Le Formal A, Verkarre V, RiouxLeclercq N, Sibony M, Bernhard JC, Camparo P, Merabet Z, Molinie V, Allory Y, Orear C, Couve S, et al. MET is a potential target across all papillary renal cell carcinomas: result from a large molecular study of pRCC with $\mathrm{CGH}$ array and matching gene expression array. Clin Cancer Res. 2014; 20:3411-3421.

36. Lefevre M, Couturier J, Sibony M, Bazille C, Boyer K, Callard P, Vieillefond A, Allory Y. Adult papillary renal tumor with oncocytic cells: clinicopathologic, immunohistochemical, and cytogenetic features of 10 cases. Am J Surg Pathol. 2005; 29:1576-1581.

37. Saleeb RM, Plant P, Tawedrous E, Krizova A, Brimo F, Evans AJ, Wala SJ, Bartlett J, Ding Q, Boles D, Rotando F, Farag M, Yousef GM. Integrated Phenotypic/Genotypic Analysis of Papillary Renal Cell Carcinoma Subtypes: Identification of Prognostic Markers, Cancer-related Pathways, and Implications for Therapy. Eur Urol Focus. 2016 Sep 22. http:// dx.doi.org/10.1016/j.euf.2016.09.002. [Epub ahead of print].

38. Kunju LP, Wojno K, Wolf JS Jr, Cheng L, Shah RB. Papillary renal cell carcinoma with oncocytic cells 
and nonoverlapping low grade nuclei: expanding the morphologic spectrum with emphasis on clinicopathologic, immunohistochemical and molecular features. Hum Pathol. 2008; 39:96-101.

39. Brunelli M, Eble JN, Zhang S, Martignoni G, Delahunt B, Cheng L. Eosinophilic and classic chromophobe renal cell carcinomas have similar frequent losses of multiple chromosomes from among chromosomes 1, 2, 6, 10, and 17 , and this pattern of genetic abnormality is not present in renal oncocytoma. Mod Pathol. 2005; 18:161-169.

40. Durinck S, Stawiski EW, Pavia-Jimenez A, Modrusan Z, Kapur P, Jaiswal BS, Zhang N, Toffessi-Tcheuyap V, Nguyen TT, Pahuja KB, Chen YJ, Saleem S, Chaudhuri $\mathrm{S}$, et al. Spectrum of diverse genomic alterations define non-clear cell renal carcinoma subtypes. Nat Genet. 2015; 47:13-21.

41. Meyer PN, Cao Y, Jacobson K, Krausz T, Flanigan RC, Picken MM. Chromosome 1 analysis in chromophobe renal cell carcinomas with tissue microarray (TMA)-facilitated fluorescence in situ hybridization (FISH) demonstrates loss of $1 \mathrm{p} / 1$ which is also present in renal oncocytomas. Diagn Mol Pathol. 2008; 17:141-144.

42. Tan MH, Wong CF, Tan HL, Yang XJ, Ditlev J, Matsuda D, Khoo SK, Sugimura J, Fujioka T, Furge KA, Kort E, Giraud S, Ferlicot S, et al. Genomic expression and single-nucleotide polymorphism profiling discriminates chromophobe renal cell carcinoma and oncocytoma. BMC Cancer. 2010; 10:196.

43. Khoury JD, Abrahams NA, Levin HS, MacLennan GT. The utility of epithelial membrane antigen and vimentin in the diagnosis of chromophobe renal cell carcinoma. Ann Diagn Pathol. 2002; 6:154-158.
44. Kolin DL, Sy K, Rotondo F, Bassily MN, Kovacs K, Brezden-Masley C, Streutker CJ, Yousef GM. Prognostic significance of human tissue kallikrein-related peptidases 6 and 10 in gastric cancer. Biol Chem. 2014; 395:1087-1093.

45. Kolin DL, Sy K, Rotondo F, Bassily MN, Kovacs K, Brezden-Masley C, Streutker CJ, Yousef GM. Prognostic significance of human tissue kallikrein-related peptidases 11 and 15 in gastric cancer. Tumour Biol. 2016; 37:437446.

46. Richard PO, Jewett MA, Bhatt JR, Kachura JR, Evans AJ, Zlotta AR, Hermanns T, Juvet T, Finelli A. Renal Tumor Biopsy for Small Renal Masses: A Single-center 13-year Experience. Eur Urol. 2015; 68:1007-1013.

47. Smaldone MC, Corcoran AT, Uzzo RG. Active surveillance of small renal masses. Nat Rev Urol. 2013; 10:266-274.

48. Kurban G, Gallie BL, Leveridge M, Evans A, Rushlow D, Matevski D, Gupta R, Finelli A, Jewett MA. Needle core biopsies provide ample material for genomic and proteomic studies of kidney cancer: observations on DNA, RNA, protein extractions and VHL mutation detection. Pathol Res Pract. 2012; 208:22-31.

49. Chen C, Ridzon DA, Broomer AJ, Zhou Z, Lee DH, Nguyen JT, Barbisin M, Xu NL, Mahuvakar VR, Andersen MR, Lao KQ, Livak KJ, Guegler KJ. Real-time quantification of microRNAs by stem-loop RT-PCR. Nucleic Acids Res. 2005; 33:e179.

50. Eisen MB, Spellman PT, Brown PO, Botstein D. Cluster analysis and display of genome-wide expression patterns. Proc Natl Acad Sci U S A. 1998; 95:14863-14868.

51. de Hoon MJ, Imoto S, Nolan J, Miyano S. Open source clustering software. Bioinformatics. 2004; 20:1453-1454. 